\title{
Cardiac Rehabilitation Increases the Reliability of the 6-Minute Walk Test in Patients After Coronary Artery Bypass Graft Surgery
}

\author{
Emruallah Hayta, ${ }^{1}$ Özge Korkmaz ${ }^{2}$ \\ ${ }^{1}$ Department of Physical Medicine and Rehabilitation, Cumhuriyet University Faculty of Medicine, Sivas, Turkey; ${ }^{2}$ Department \\ of Cardiovascular Surgery, Cumhuriyet University Faculty of Medicine, Sivas, Turkey
}

\section{ABSTRACT}

Background: Few data are available on the capacity for functional exercise after cardiac rehabilitation in patients who have undergone coronary artery bypass graft (CABG) surgery. The aim of this study was to determine the clinical and biochemical factors that affect the 6-minute walk test (6MWT) results in patients who have undergone CABG.

Methods: Data were prospectively collected from 56 patients consecutively admitted at our hospital between January 2013 and May 2015 for a 3-month cardiac rehabilitation program. Data were analysed retrospectively up to 90 days after cardiac surgery. In addition to clinical and biochemical parameters, the functional capacity of patients was evaluated by an exercise 6-minute walking test and echocardiography. Functional capacity was evaluated at baseline and after three months of cardiac rehabilitation.

Results: Before cardiac rehabilitation, the 6MWT values were negatively correlated with Body Mass Index (BMI) and abdominal and buttock diameters $(\mathrm{r}=-0.375,-0.386$, and -0.370 , respectively; $P<.05$ ), and were positively correlated with metabolic equivalent (MET) values $(\mathrm{r}=0.493, P<.05)$. After cardiac rehabilitation, the 6MWT values were negatively correlated with body mass index (BMI) and abdominal and buttock diameters $(\mathrm{r}=-0.382,-0.274$, and -0.405 , respectively; $P<.05)$ and were positively correlated with MET and $\mathrm{VO}_{2}$ max values $(\mathrm{r}=0.456$ and 0.573 , respectively; $P<0.05)$. Before cardiac rehabilitation, $\mathrm{VO}_{2}$ max and FEV1/FEVC values were found as factors that significantly increased 6MWT values $(P<.05)$. After cardiac rehabilitation, $\mathrm{VO}_{2}$ max values were found as factors that significantly increased 6MWT values $(P<.05)$.

Conclusion: Overall, in current clinical settings, cardiac rehabilitation increases the reliability of the 6MWT. Improvement in pulmonary function after cardiac rehabilitation reduces the impact of pulmonary function on 6MWT values. The functional capacity of patients may be more reliably determined by $6 \mathrm{MWT}$ after cardiac rehabilitation.

Received December 18, 2016; received in revised form October 2, 2017; accepted October 25, 2017.

Correspondence: Dr. Emrullah Hayta, Department of Physical Medicine and Rehabilitation, Cumburiyet University Faculty of Medicine, 58140 Sivas, Turkey; +346 2580000; (e-mail: dremay@gmail.com).

\section{INTRODUCTION}

Cardiac rehabilitation, a branch of rehabilitation medicine, is an important component of the multidisciplinary management of patients after coronary artery bypass graft (CABG) surgery [Strong 2012]. Cardiac rehabilitation involves exercise training, patient education, risk reduction counselling, and interventions pertaining to behavior and lifestyle. Patients who are suitable candidates for cardiac rehabilitation are usually able to start a program within 6 weeks after CABG [Kulik 2016]. The duration of the program varies from patient-topatient and can range from six months to several years. Even after a cardiac rehabilitation program is finished, there are long-term maintenance programs available, as the benefits of rehabilitation are maintained only with long-term adherence [King 2012; Goel 2015].

Cardiac rehabilitation aims to improve the physiologic and psychosocial condition of patients. The physiologic benefits include an increased exercise capacity and reduction of risk factors - smoking cessation, reduction in body weight, blood pressure, and other risk parameters, including the risk and progression of atherosclerosis [Parmley 1986; Fu 2014]. Psychological improvements include a reduction in depression, anxiety and stress. The sum total of these improvements enable the patient to acquire and maintain functional independence and to return to activities that benefit both the patient and their families, and society in general [McKee 2009].

The measurement of exercise capacity is integral to the assessment of patients with cardiopulmonary disease. The 6-min walk test $(6 \mathrm{MWT})$ provides information regarding functional capacity, response to therapy, and prognosis across a range of chronic cardiopulmonary conditions [Rasekaba 2009; Bellet 2012]. In a 2000 study, Hamilton and colleagues found the $6 \mathrm{MWT}$ to be a reliable test for assessment of functional capacity in a Phase II/III cardiac rehabilitation population [Hamilton 2000]. The 6MWT is a useful measure of functional capacity targeted at patients with a minimum of moderately severe impairment. The test has been widely used for preoperative and postoperative evaluation and for measuring the response to therapeutic interventions for pulmonary and cardiac disease [Cacau 2016].

In a review of literature on cardiac rehabilitation after $\mathrm{CABG}$, Mendes et al. found there was not enough evidence to draw reliable conclusions about the impact of cardiac rehabilitation on patient outcome [Cacciatore 2012; Mendes 2016]. Although the 6MWT has been used successfully by 
other investigators to assess functional status in a variety of patient populations, intra- and inter-rater reliability of the $6 \mathrm{MWT}$ and its validity in patients undergoing cardiac rehabilitation requires further research [Wright 2001; Haeffener 2008]. The aim of this study was to determine the clinical and biochemical parameters that can affect the results of the 6-minute walk test (6MWT) results in patients who have undergone CABG.

\section{MATERIALS AND METHODS}

In the present study, 118 patients who underwent CABG surgery between January 2013 and May 2015 in the Department of Cardiovascular Surgery at our hospital and subsequently enrolled in a cardiac rehabilitation program were retrospectively assessed for eligibility for the study. Patients with musculoskeletal disorders, end-stage chronic obstructive pulmonary disease, or decompensated heart failure were excluded from the study, since these can affect 6MWT performance. Fifty-six patients were found eligible and enrolled in the study, and their clinical data was used for the statistical evaluation. Patient age, BMI, abdominal and buttock diameters, blood lipids, insulin and thyroid levels, and echocardiography findings pre- and post cardiac rehabilitation were collected from the patient information system of the Cardiac Rehabilitation Unit of our hospital for data analysis.

$\mathrm{VO}_{2}$ max and MET Calculations with Cardiopulmonary Tests

Measurements were taken using a SFT Care Fusion ergospirometry device (Germany). The tests were repeated until the difference between the three tests was $10 \%$, or when the value determined automatically by Care Fusion ergospirometry device was recorded as the best value. A breath by breath analysis system was used to measure $\mathrm{VO}_{2}$ max. Fusion ergospirometry was used to measure $\mathrm{VO}_{2}, \mathrm{VCO}_{2}$ and the volume of expired air per minute (VE). $\mathrm{ME}^{2} \mathrm{~T}$ was determined at the end of the test (MET maximum) by the ratio: MET maximum $=\mathrm{VO}_{2} \max \left(\mathrm{m} \cdot \mathrm{kg}^{-1} \cdot \mathrm{min}^{-1}\right) / \mathrm{VO}_{2}$ rest $\left(\mathrm{ml} \cdot \mathrm{kg}^{-1} \cdot \mathrm{min}^{-1}\right)[$ Ainsworth 2000].

\section{Six Minute Walk Test (6MW'T)}

The 6MWT was developed by the American Thoracic Society (ATS). Subjects walked at their own maximum pace along a flat a 30 meter track, aiming to cover as much distance as possible in the timed 6 minutes. Their distance walked was recorded in meters [ATS Committee 2002].

\section{Body Fat Ratio Measurement}

Bioelectric impedance measurements were taken on patients with an empty stomach and bladder in the morning after a minimum 8 hours of sleep. A Tanita Body Composition Analyser TBF 300 was used for the measurements. Hip circumference was measured on the line passing through most protruding point of gluteus maximus muscle and pubis.

\section{Blood Samples}

Blood samples were taken in the morning following at least 12 hours of overnight fasting. TK, HDL cholesterol and triglyceride levels were analysed using an Olympus AU 5223 analyser. Insulin was analysed once a day using electrochemiluminescence immunoassay (ECLIA). Chemiluminescence enzyme immunometric assay was used for thyroid function analysis.

\section{Implementation of Exercise Programs}

Individual exercise prescriptions were formulated based on cardiopulmonary test results. The aerobic exercise program consisted of 3 days of exercise per week for 12 weeks with a combination of fast walking and low-intensity running. Exercise intensity was calculated using the Karvonen formula, taking into account the maximal heart rate (MHR) reached during an exercise tolerance test. A 5 minute warm up phase preceded a 30-50 minute exercise phase, followed by a $5-10$ minute cooling period. Aerobic exercises were implemented every other day, on three predefined days a week.

Table 1. Selected clinical parameters assessed before and after cardiac rehabilitation.

\begin{tabular}{|c|c|c|c|c|}
\hline & Before & After & Difference & $p$ \\
\hline Weight (kg) & $97.40 \pm 16.4$ & $91.2 \pm 14.4$ & $6.2 \pm 6.12$ & 0.001 \\
\hline $\mathrm{BMI}\left(\mathrm{kg} / \mathrm{m}^{2}\right)$ & $36.7 \pm 6.6$ & $34.15 \pm 6.1$ & $2.6 \pm 2.3$ & 0.001 \\
\hline Abdominal diameter $(\mathrm{cm})$ & $114.3 \pm 1.5$ & $106.4 \pm 14.3$ & $7.9 \pm 4.6$ & 0.001 \\
\hline Buttock diameter $(\mathrm{cm})$ & $118.9 \pm 15.3$ & $112.8 \pm 12.3$ & $6.0 \pm 4.9$ & 0.001 \\
\hline Thigh diameter $(\mathrm{cm})$ & $58.4 \pm 7.9$ & $55.4 \pm 7.7$ & $3.0 \pm 3.6$ & 0.001 \\
\hline Body fat ratio (\%) & $39.5 \pm 8.4$ & $35.2 \pm 8.2$ & $4.1 \pm 4.0$ & 0.001 \\
\hline Cholesterol (mg/dL) & $203.5 \pm 65.6$ & $178.8 \pm 47.0$ & $23.6 \pm 66.3$ & 0.024 \\
\hline Triglyceride $(\mathrm{mg} / \mathrm{dL})$ & $205.8 \pm 159.1$ & $179.3 \pm 167.5$ & $26.5 \pm 82.3$ & 0.034 \\
\hline $\mathrm{LDL}(\mathrm{mg} / \mathrm{dL})$ & $125.8 \pm 36.9$ & $110.0 \pm 31.6$ & $15.7 \pm 31.8$ & 0.002 \\
\hline $\mathrm{HDL}(\mathrm{mg} / \mathrm{dL})$ & $39.3 \pm 9.6$ & $41.8 \pm 10.2$ & $2.6 \pm 9.1$ & 0.059 \\
\hline T3 (pmol/L) & $2.9 \pm 0.5$ & $2.8 \pm 0.5$ & $0.1 \pm 0.5$ & 0.427 \\
\hline $\mathrm{T} 4$ (pmol/L) & $1.1 \pm 0.4$ & $1.0 \pm 0.2$ & $0.1 \pm 0.3$ & 0.128 \\
\hline $\mathrm{TSH}(\mathrm{pmol} / \mathrm{L})$ & $1.6 \pm 1.0$ & $1.4 \pm 0.9$ & $0.01 \pm 0.5$ & 0.843 \\
\hline Insulin $(I U / m L)$ & $20.1 \pm 11.6$ & $13.5 \pm 6.8$ & $6.6 \pm 7.8$ & 0.001 \\
\hline FEV1(\%) & $92.4 \pm 16.7$ & $97.8 \pm 16.3$ & $4.9 \pm 8.7$ & 0.001 \\
\hline FVC (\%) & $86.1 \pm 17.5$ & $90.9 \pm 16.6$ & $5.4 \pm 8.4$ & 0.001 \\
\hline FEV1/FVC (\%) & $78.3 \pm 10.8$ & $79.4 \pm 10.0$ & $0.8 \pm 4.9$ & 0.391 \\
\hline $\mathrm{EF}(\%)$ & $55.3 \pm 6.9$ & $56.4 \pm 7.7$ & $1.4 \pm 3.3$ & 0.005 \\
\hline 6MWT (m) & $448.8 \pm 170.8$ & $554.2 \pm 148.7$ & $-92.53 \pm 8.7$ & 0.001 \\
\hline MET & $5.1 \pm 1.1$ & $6.3 \pm 1.1$ & $1.2 \pm 0.7$ & 0.001 \\
\hline $\mathrm{VO}_{2} \max (\mathrm{ml} / \mathrm{kg} / \mathrm{min})$ & $19.7 \pm 4.1$ & $22.7 \pm 3.8$ & $2.9 \pm 2.3$ & 0.001 \\
\hline
\end{tabular}

Data were presented as mean \pm SD.

BMI, body mass index; LDL, low density lipoprotein; HDL, high density lipoprotein; TSH, thyroid stimulating hormone; FEV1, forced expiratory volume; FVC, forced vital capacity; EF, ejection fraction; 6MWT, 6-min walk test; MET, metabolic equivalent; $\mathrm{VO}_{2}$ max, maximum rate of oxygen consumption. 


\section{STATISTICAL ANALYSIS}

Clinical data of the study group were presented as mean $\pm \mathrm{SD}$ or percentage. For analyses of the clinical data, IBM SPSS Statistics for Windows, Version 22.0 (IBM Corp, Armonk, NY, USA) was used. In the assessment of normality, Kolmogorov-Smirnov test was used. Paired t and Spearman correlation tests were used to assess clinical variables. With multiple regression analysis, with the help of multicollinearity to ignore unsuitable variables, we determined the variables that had a meaningful effect on the $6 \mathrm{MWT}$ values. A $P$ value of less than .05 was accepted as significant.

\section{RESULTS}

Data analysis of 56 cardiac rehabilitation patients in the study group had a male/female ratio of $24(52.2 \%) / 22(47.8 \%)$,

Table 2. Correlation of 6MWT value with clinical parameters before and after cardiac rehabilitation.

\begin{tabular}{|c|c|c|c|c|}
\hline \multirow[b]{2}{*}{ Baseline Value } & \multicolumn{2}{|c|}{ Before } & \multicolumn{2}{|c|}{ After } \\
\hline & r & $p$ & r & $p$ \\
\hline Age $(y)$ & -0.183 & 0.225 & -0.183 & 0.225 \\
\hline $\mathrm{BMI}\left(\mathrm{kg} / \mathrm{m}^{2}\right)$ & -0.375 & 0.010 & -0.382 & 0.004 \\
\hline Abdominal diameter $(\mathrm{cm})$ & -0.386 & 0.008 & -0.274 & 0.033 \\
\hline Buttock diameter $(\mathrm{cm})$ & -0.370 & 0.011 & -0.405 & 0.003 \\
\hline Thigh diameter $(\mathrm{cm})$ & -0.242 & 0.105 & -0.176 & 0.121 \\
\hline Body fat ratio (\%) & -0.278 & 0.071 & 0.431 & 0.002 \\
\hline Cholesterol (mg/dL) & -0.180 & 0.236 & -0.170 & 0.136 \\
\hline Triglyceride (mg/dL) & -0.044 & 0.770 & -0.005 & 0.487 \\
\hline $\mathrm{LDL}(\mathrm{mg} / \mathrm{dL})$ & -0.095 & 0.531 & -0.126 & 0.202 \\
\hline $\mathrm{HDL}(\mathrm{mg} / \mathrm{dL})$ & -0.153 & 0.309 & -0.229 & 0.063 \\
\hline T3 (pmol/L) & 0.085 & 0.601 & -0.090 & 0.279 \\
\hline $\mathrm{T} 4$ (pmol/L) & 0.045 & 0.783 & 0.162 & 0.173 \\
\hline $\mathrm{TSH}(\mathrm{pmol} / \mathrm{L})$ & 0.001 & 0.994 & 0.046 & 0.394 \\
\hline Insulin (IU/mL) & 0.207 & 0.195 & -0.011 & 0.474 \\
\hline FVC (\%) & 0.119 & 0.453 & 0.008 & 0.479 \\
\hline FEV (\%) & 0.260 & 0.096 & 0.011 & 0.471 \\
\hline FEV1/FVC (\%) & -0.156 & 0.350 & 0.153 & 0.198 \\
\hline MET (metabolic equivalent) & 0.493 & 0.001 & 0.456 & 0.001 \\
\hline $\mathrm{VO}_{2} \max (\mathrm{ml} / \mathrm{kg} / \mathrm{min})$ & 0.297 & 0.05 & 0.573 & 0.001 \\
\hline $\mathrm{EF}(\%)$ & -0.060 & 0.692 & -0.074 & 0.316 \\
\hline
\end{tabular}

Data were presented as mean \pm SD.

BMI, body mass index; LDL, low density lipoprotein; HDL, high density lipoprotein; TSH, thyroid stimulating hormone; FEV1, forced expiratory volume; FVC, forced vital capacity; EF, ejection fraction; 6MWT, 6-min walk test; MET, metabolic equivalent; $\mathrm{VO}_{2}$ max, maximum rate of oxygen consumption. and a mean age of $57.21 \pm 5.34$ years. Table 1 lists the clinical parameters measured before and after cardiac rehabilitation. Weight, BMI, abdominal, buttock, and thigh diameters, body fat ratio, cholesterol, triglyceride, LDL, and insulin levels were all significantly lower after cardiac rehabilitation compared to before cardiac rehabilitation $(P<.05)$. HDL levels, FEV1, FVC, FEV1/FVC and EF ratios, 6MWT, MET, and VO2 max were all significantly higher after cardiac rehabilitation than before cardiac rehabilitation $(P<.05)$. There was no significant change in the T3, T4, and TSH levels and FEV1/ FVC values before and after cardiac rehabilitation $(P>.05)$.

Table 2 shows the correlation between 6MWT values and clinical parameters before and after cardiac rehabilitation. Before cardiac rehabilitation, $6 \mathrm{MWT}$ values were significantly negatively correlated with BMI and abdominal and buttock diameters $(\mathrm{r}=-0.375,-0.386$, and -0.370 , respectively; $P<.05)$. Before cardiac rehabilitation, the $6 \mathrm{MWT}$ values were significantly positively correlated with MET values $(r=0.493, P<.05)$. After cardiac rehabilitation, the $6 M W T$ values were significantly negatively correlated with BMI and abdominal and buttock diameters $(\mathrm{r}=-0.382$, -0.274 , and -0.405 , respectively; $P<.05$ ). After cardiac rehabilitation, the $6 \mathrm{MWT}$ values were significantly positively correlated with MET and $\mathrm{VO}_{2}$ max values $(\mathrm{r}=0.456$ and 0.573 , respectively; $P<.05)$.

Table 3 shows the results of multiple regression analyses of clinical variables that significantly affected $6 \mathrm{MWT}$ values before and after cardiac rehabilitation. Before cardiac rehabilitation, with the regression model, the factors affecting the results of $6 \mathrm{MWT}$ values can be predicted with a higher percentage $(92.4 \%)\left(\mathrm{R}^{2}=0.924\right)$. Before cardiac rehabilitation, $\mathrm{VO}$, max and FEV1/FEVC values were found to be factors that significantly increased the $6 \mathrm{MWT}$ values $(P<.05)$. After cardiac rehabilitation, with the regression model, the factors affecting the results of $6 \mathrm{MWT}$ values can be predicted with a higher percentage $(95.6 \%)\left(\mathrm{R}^{2}=0.956\right)$. After cardiac rehabilitation, $\mathrm{VO}_{2}$ max values were found as factors that significantly increased the $6 \mathrm{MWT}$ values $(P<.05)$. According to the results of multiple regression analyses, other clinical parameters did not have a meaningful contribution to the results of the $6 \mathrm{MWT}$.

Table 3. Results of multiple regression analyses: clinical variables affecting the 6MWT value before and after cardiac rehabilitation.

\begin{tabular}{|c|c|c|c|c|c|c|}
\hline & & \multicolumn{2}{|c|}{$\begin{array}{c}\text { Unstandardized } \\
\text { Coefficients }\end{array}$} & \multicolumn{3}{|c|}{$\begin{array}{l}\text { Standardized } \\
\text { Coefficients }\end{array}$} \\
\hline & & B & Std. Error & Beta & $\mathrm{t}$ & Sig. \\
\hline \multirow[t]{2}{*}{ Before } & $\mathrm{VO}_{2} \max$ & 10.315 & 4.990 & 0.440 & 2.067 & 0.046 \\
\hline & FEV1/FEVC & 3.166 & 1.279 & 0.527 & 2.475 & 0.018 \\
\hline After & $\mathrm{VO}_{2} \max$ & 24.558 & 0.824 & 0.978 & 29.788 & 0.001 \\
\hline
\end{tabular}

FEV1, Forced expiratory volume; FVC, Forced vital capacity; $\mathrm{VO}_{2}$ max, Maximum rate of oxygen consumption. 


\section{DISCUSSION}

To our knowledge, there have been no prior studies to assess the clinical factors that can potentially impact 6MWT performance in subjects after a 3 -month cardiac rehabilitation in patients who have undergone CABG surgery. Our findings showed that cardiac rehabilitation improves blood lipid parameters, pulmonary function test values, and the capacity for physical activity, a finding supported by some previous studies [Anderson 2016]. Before cardiac rehabilitation, the 6MWT values had a weak negative association with obesity, and a weak positive association with exercise capacity. After cardiac rehabilitation, the 6MWT values had a weak negative association with the obesity parameters, although it had a positive association with exercise capacity and maximum oxygen consumption. Multiple regression analyses revealed that before cardiac rehabilitation, the maximum rate of oxygen consumption and exercise capacity had meaningful effects on 6MWT values, and after cardiac rehabilitation, only the maximum rate of oxygen consumption had an effect on $6 \mathrm{MWT}$ values. Our findings suggest that the pulmonary function test values were significantly increased after cardiac rehabilitation, and this increase had a positive affect on other pulmonary function parameters and at the end of the rehabilitation period. However, their effects on the 6MWT values were lost after cardiac rehabilitation ended. Overall, the $6 \mathrm{MWT}$ can be used more reliably to assess the functional capacity of patients after cardiac rehabilitation than if used before cardiac rehabilitation.

CABG is currently performed in about $11 \%$ of patients with acute coronary syndrome and complex coronary anatomy (defined by a Syntax score $>22$ ) and significant comorbidities [Yerokun 2016]. The Cardiac Rehabilitation Outcome Study (CROS) [Rauch 2016] assessed the prognostic effect of cardiac rehabilitation in patients, including those with a history of CABG. The authors concluded that cardiac rehabilitation likely contributes to reduced mortality. However, they caution that there needs to be a consensus on the standards applied in its application in patients with specific cardiac disorders.

We think that the success of cardiac rehabilitation, as measured by an improvement in 6MWT results, indicates an increase in a patient's capacity for physical activity. Moreover, a reduced BMI and beneficial modifications of other clinical parameters can make important contributions to the general health and quality of life of patients. In a recent metaanalysis of patients with coronary artery disease, physical performance testing was found to be a quick, safe, inexpensive, and reliable assessment tool for determining clinical status and designing treatment protocols [Yamamoto 2016]. In another study, Fiorina et al. evaluated the use of 6MWT to assess the success of cardiac rehabilitation programs and examined the effect of several clinical variables on the 6MWT performance of patients. They found that $6 \mathrm{MWT}$ results can be useful as reference values for distance walked after CABG and for the early clinical assessment of patients after cardiac surgery [Fiorina 2007]. Our findings show that the use of $6 \mathrm{MWT}$ can have some limitations in patients without optimal pulmonary function capacity when assessed in early weeks after CABG surgery.

Over the past 30 years of research, cardiac rehabilitation has been reported in the literature as an established approach in the management of cardiovascular diseases with variable short- and long-term success [Højskov 2016]. Cardiac rehabilitation programs need to be standardized with regard to content, application, and evaluation of its effects on clinical parameters, according to subtypes of cardiovascular diseases. This will increase its acceptance and adoption by both physicians and patients [Pack 2013; Haykowsky 2016].

Our main objective was to determine the clinical and biochemical factors that affect the 6MWT results at the end of a 3 -month cardiac rehabilitation period in patients who have undergone $\mathrm{CABG}$. We conclude that in patients after $\mathrm{CABG}$ surgery, cardiac rehabilitation has significant potential to improve the health and quality of life of patients in the short-term.

Our study has some limitations. Follow-up duration was 3 months. With our data we cannot determine whether poorer 6MWT and lower physical activity levels are associated with increased unfavorable events over a longer-term follow-up. Because of the observational study design, there may be residual confounding by unmeasured variables. Our findings also might not be generalizable to patients who are unwilling or unable to complete all procedures in study visits.

The 6MWT and the measures of physical activity reported here can be obtained relatively quickly and for minimal cost, and can provide important reliable information about the clinical benefits of cardiac rehabilitation in patients after CABG surgery. Overall, after cardiac rehabilitation, the reliability of $6 \mathrm{MWT}$ increases compared to its use before cardiac rehabilitation. Improvement of pulmonary function after cardiac rehabilitation reduces the interaction of pulmonary function parameters on the 6MWT values, and so the functional capacity of patients may be more reliably determined by $6 \mathrm{MWT}$ after cardiac rehabilitation. Because the $6 \mathrm{MWT}$ performance and physical activity levels in patients after CABG surgery can be improved with exercise interventions, further study is needed to determine the amount of intervention that can improve the $6 \mathrm{MWT}$ results in the long-term outcome of patients after CABG surgery.

\section{REFERENCES}

Ainsworth BE, Haskell WL, Whitt MC, et al. 2000. Compendium of physical activities: an update of activity codes and MET intensities. Med Sci Sports Exerc 32(9 Suppl):498-504.

Anderson L, Oldridge N, Thompson DR, et al. 2016. Exercise-Based Cardiac Rehabilitation for Coronary Heart Disease: Cochrane Systematic Review and Meta-Analysis. J Am Coll Cardiol 67:1-12.

ATS Committee on Proficiency Standards for Clinical Pulmonary Function Laboratories. 2002. ATS statement: guidelines for the six-minute walk test. Am J Respir Crit Care Med 166:111-117.

Bellet RN, Adams L, Morris NR. 2012. The 6-minute walk test in outpatient cardiac rehabilitation: validity, reliability and responsiveness a systematic review. Physiotherapy 98:277-286.

Cacau LA, Santana-Filho VJ, Maynard LG, et al. 2016. Reference Values 
for the Six-Minute Walk Test in Healthy Children and Adolescents: a Systematic Review. Braz J Cardiovasc Surg 31:381-388.

Cacciatore F, Abete P, Mazzella F, et al. 2012. Six-minute walking test but not ejection fraction predicts mortality in elderly patients undergoing cardiac rehabilitation following coronary artery bypass grafting. Eur J Prev Cardiol 19:1401-1409.

Fiorina C1, Vizzardi E, Lorusso R, et al. 2007. The 6-min walking test early after cardiac surgery. Reference values and the effects of rehabilitation programme. Eur J Cardiothorac Surg 32:724-729.

Fu TC, Huang SC, Hsu CC, et al. 2014. Cardiac Rehabilitation in Patients with Heart Failure. Acta Cardiol Sin 30:353-359.

Goel K, Pack QR, Lahr B, et al. 2015. Cardiac rehabilitation is associated with reduced long-term mortality in patients undergoing combined heart valve and CABG surgery. Eur J Prev Cardiol 22:159-168.

Haeffener MP, Ferreira GM, Barreto SS, et al. 2008. Incentive spirometry with expiratory positive airway pressure reduces pulmonary complications, improves pulmonary function and 6-minute walk distance in patients undergoing coronary artery bypass graft surgery. Am Heart J 156:900.e1-900.e8.

Hamilton DM, Haennel RG. 2000. Validity and reliability of the 6-minute walk test in cardiac rehabilitation population. J Cardiopulm Rehabil 20:156-164.

Haykowsky MJ, Daniel KM, Bhella PS, et al. 2016. Heart Failure: Exercise-Based Cardiac Rehabilitation: Who, When, and How Intense? Can J Cardiol 32:382-387.

Højskov IE, Moons P, Hansen NV, et al. 2016. Early physical training and psycho-educational intervention for patients undergoing coronary artery bypass grafting. The SheppHeart randomized $2 \times 2$ factorial clinical pilot trial. Eur J Cardiovasc Nurs 15:425-437.

King M, Bittner V, Josephson R, et al. 2012. Medical director responsibilities for outpatient cardiac rehabilitation/secondary prevention programs: 2012 update: a statement for health care professionals from the American Association for Cardiovascular and Pulmonary Rehabilitation and the American Heart Association. J Cardiopulm Rehabil Prev $32: 410-419$.
Kulik A. 2016. Secondary prevention after coronary artery bypass graft surgery: a primer. Curr Opin Cardiol 31:635-643.

McKee G. 2009. Are there meaningful longitudinal changes in health related quality of life-SF36, in cardiac rehabilitation patients? Eur J Cardiovasc Nurs 8:40-47.

Mendes M. 2016. Is There a Role for Cardiac Rehabilitation After Coronary Artery Bypass Grafting? There is No Role for Cardiac Rehabilitation After Coronary Artery Bypass Grafting. Circulation 133:2538-2543.

Pack QR, Goel K, Lahr BD, et al. 2013. Participation in cardiac rehabilitation and survival after coronary artery bypass graft surgery: a community-based study. Circulation 128:590-597.

Parmley WW. 1986. Position report on cardiac rehabilitation: Recommendations of the American College of Cardiology. J Am Coll Cardiol 7:451-453.

Rasekaba T, Lee AL, Naughton MT, et al. 2009. The six-minute walk test: a useful metric for the cardiopulmonary patient. Intern Med J 39:495-501.

Rauch B, Davos CH, Doherty P, et al. 2016. The prognostic effect of cardiac rehabilitation in the era of acute revascularisation and statin therapy: A systematic review and meta-analysis of randomized and non-randomized studies - The Cardiac Rehabilitation Outcome Study (CROS). Eur J Prev Cardiol 23:1914-1939.

Strong PC, Lee SH, Chou YC, et al. 2012. Relationship between quality of life and aerobic capacity of patients entering phase II cardiac rehabilitation after coronary artery bypass graft surgery. J Chin Med Assoc 75:121-126.

Wright DJ, Khan KM, Gossage EM, et al. 2001. Assessment of a lowintensity cardiac rehabilitation programme using the six-minute walk test. Clin Rehabil 15:119-124.

Yamamoto S, Yamaga T, Sakai Y, et al. 2016. Association between physical performance and cardiovascular events in patients with coronary artery disease: protocol for a meta-analysis. Syst Rev 5:32.

Yerokun BA, Williams JB, Gaca J, et al. 2016. Indications, algorithms, and outcomes for coronary artery bypass surgery in patients with acute coronary syndromes. Coron Artery Dis 27:319-326. 